\title{
Thickness Dependence of Surface Wettability Change by Photoreactive Polymer Nanosheets
}

\author{
By Sabiha SultanA, ${ }^{1}$ Jun MATSUI, ${ }^{1,2, *}$ Masaya MitSUISHI,${ }^{1}$ and Tokuji MiYASHITA ${ }^{1, *}$
}

This paper describes the photochemical surface modification of a solid substrate using a photoreactive polymer nanosheets. A neo-pentylmethacrylamide-co-phenylmethacrylamide ( $\mathrm{p}(n \mathrm{PMA} / \mathrm{PhMA})$ ), which has been reported to form a stable monolayer was transferred onto a solid substrate using the Langmuir-Blodgett (LB) technique. The $\mathrm{p}(n \mathrm{PMA} / \mathrm{PhMA})$ copolymer was deposited with different number of layers onto a solid substrate. Then the wettability change after deep UVirradiation was observed. The water contact angle (CA) of a substrate coated with $\mathrm{p}(n P \mathrm{MA} / \mathrm{PhMA})$ nanosheet initially shows a value of $80^{\circ}$ attributed to alkyl and phenyl side chains of $\mathrm{p}(n \mathrm{PMA} / \mathrm{PhMA})$. After 30 min irradiation, the water CA of the surface decreases to $14^{\circ}$ because of formation of carbonyl groups (COO) of ketone, aldehyde and carboxylic acid from photodecomposition of the nanosheet. The decrease in contact angle depends on number of deposited layers: a thicker film implies smaller surface CA. Results of UV-vis spectroscopy, Fourier transform infrared spectroscopy (FT-IR), and X-ray photoelectron spectrscopy (XPS) suggest that more numerous nanosheets produce more oxygen containing species after deep UV irradiation, which implies that the number of the deposited nanosheets can control surface wettability.

KEY WORDS: Polymer Nanosheet / Surface Wettability / Photoreaction / Nanocoating /

The wettability of solid surfaces is an important physical property that can be described by a combination of chemistry and physics, i.e., the chemical composition and the geometrical microstructure of the surface. ${ }^{1-3}$ Recently, tailor-made control of surface wettability has become an active area of research because of its anticipated application to micro-devices, such as microfluidic devices and controllable separation system. ${ }^{4-7}$

Wettability of a solid substrate can be tuned using ultrathin films such as self-assembly monolayer, ${ }^{8}$ layer-by-layer assembled film ${ }^{9}$ and Langmuir-Blodgett (LB) film. ${ }^{8}$ The LB technique enables to prepare closed packed and highly oriented film with fine control at nanoscale thickness. We have reported that several acrylamide derivative polymers form a highly oriented polymer monolayer on a water surface. ${ }^{10}$ The polymers form a densely packed and stable polymer monolayer (polymer nanosheet) because of a two-dimensional hydrogen bonding network that exist between the amide groups. Several kinds of polymer nanosheets were used for modifying surface wettability. ${ }^{11,12}$ For example, fluorinated polymer nanosheets was used to produce low energy surface. A substrate coated with the fluorinated polymer nanosheets shows very low critical surface tensions $(9.2 \mathrm{mN} / \mathrm{m})$ because of the close packing of the fluoroalkyl chains. ${ }^{13}$

The surface covered with ultrathin films can be changed from hydrophobic to hydrophilic using a photodecomposition of the ultrathin films. ${ }^{14-18}$ For example, Sugimura et al. ${ }^{16,17}$ and Takahara et al. ${ }^{18}$ irradiated vacuum ultraviolet light to alkyland fluoroalkylsilane SAMs to carry out photodecomposition of the SAMs. The irradiated part was decomposed to form Si$\mathrm{OH}$ group and became hydrophilic. On the other hand, we have reported a water developable positive-tone photoresist using a photoreactive polymer nanosheet; poly(neo-pentylmethacrylamide-co-phenylmethacrylamide), ( $\mathrm{p}(n \mathrm{PMA} / \mathrm{PhMA}) .{ }^{19}$ The photoirradiated part of the nanosheet becomes soluble to hot water, which suggest that the photoirradiated part was decomposed to be hydrophilic. However, details of wettability change after photoirradiation and its process were not reported. In this paper, the effect of irradiation time and film thickness to the contact angle change was reported. Interestingly, we have found that not only the irradiation time, but also the film thickness effects the wettability change after photoirradiation process. The result was characterized using spectroscopic and microscopic measurements.

\section{EXPERIMENTAL}

Synthesis of $\mathrm{p}(n \mathrm{PMA} / \mathrm{PhMA})$ (Figure 1) has been described elsewhere. ${ }^{19}$ A copolymer with $20 \mathrm{~mol} \%$ of PhMA content was used.

Surface pressure $(\pi)$-Area (A) isotherm measurement and the deposition of a $\mathrm{p}(n \mathrm{PMA} / \mathrm{PhMA})$ monolayer were carried out using an automatically functioning Langmuir trough (FSD50 and 51; USI). Distilled and deionized water $(>17.5 \mathrm{M} \Omega \mathrm{cm}$, CPW-101; Advantec) was used as the subphase. The copolymer monolayer was compressed at a rate of $15.0 \mathrm{~cm}^{2} / \mathrm{min}$. The copolymer monolayer was transferred onto solid substrates using a vertical dipping mode at a dipping speed of $10 \mathrm{~mm} / \mathrm{min}$ under surface pressure of $20 \mathrm{mN} / \mathrm{m}$ at $15^{\circ} \mathrm{C}$. The polymer nanosheets were transferred onto solid substrate as Y-type LB films with a transfer ratio of almost unity. The quartz and

\footnotetext{
${ }^{1}$ Institute of Multidisciplinary Research for Advanced Materials (IMRAM), Tohoku University, Katahira 2-1-1, Aoba-ku, Sendai 980-8577, Japan

${ }^{2}$ Precursory Research for Embryonic Science and Technology (PRESTO) Japan Science and Technology Agency, 4-1-8, Honcho, Kawaguchi 332-001

*To whom correspondence should be addressed (Tel: +81-22-217-5639, Fax: +81-22-217-5639, E-mail: jun_m@ tagen.tohoku.ac.jp (J. M), Tel: +81-22217-5637, Fax: +81-22-217-5642, E-mail: miya@tagen.tohoku.ac.jp (T. M)).
} 


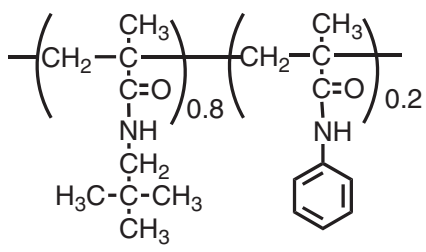

Figure 1. Chemical structure of poly(neo-pentylmethacrylamide-co-phenylmethacrylamide).

silicon substrates on which the nanosheet was deposited were cleaned by treatment with a UV-O 3 cleaner (NL-UV253; Nippon Laser and Electronics Laboratory) and were made hydrophobic by immersion of the substrates into a $c a .1 \times$ $10^{-6} \mathrm{M}$ octadecyltrichlorosilane (Shinetsu Chemical Co.) chloroform solution.

The number average molecular weight $\left(M_{\mathrm{n}}\right)$ and polydispersity index $\left(M_{\mathrm{w}} / M_{\mathrm{n}}\right)$ were determined using a gel permeation chromatograph (GPC; Tosoh Corp.) with a polystyrene standard. The molecular weights and polydispersities for $\mathrm{p}(n \mathrm{PMA} /$ PhMA) were $9.34 \times 10^{3}$ and 1.75 respectively. The UV absorption measurements were carried out using a UV-vis spectrophotometer (U-3000; Hitachi Ltd.). The contact angle (CA) measurements were carried out using a CA-X contact angle meter (Kyowa Interface Science Co. Ltd.). The CA on the surface of polymer nanosheets was measured at $3 \mathrm{~min}$ after dropping distilled water using the sessile drop method at $20^{\circ} \mathrm{C}$ and ambient humidity. The $\mathrm{CA}$ was the average of four measurements at different positions for one sample; the volume of water drops was ca. $50 \mu$ l. The FT-IR spectra were measured using a spectrometer (FT-IR-230; Jasco Inc.). As a substrate for FT-IR measurements, $\mathrm{CaF}_{2}$ was used. The $\mathrm{p}(n \mathrm{PMA} /$ PhMA) nanosheet was irradiated using a deep UV lamp (UXM501; Ushio Inc.) through a water filter $\left(61 \mathrm{~mW} / \mathrm{cm}^{2}\right)$ in air. An X-ray photoelectron spectrometer (ESCA-200; Scienta K.K.) was used to obtain XPS spectra. It was operated at $15 \mathrm{kV}$ and $26.2 \mathrm{~mA}$ with an Al-K $\alpha$ X-ray source. The take-off angle was fixed at $45^{\circ}$. All binding energies in XPS measurements were referenced to the $\mathrm{C} 1 s$ peak for neutral carbon, which was assigned as a value of $285.0 \mathrm{eV}$. Atomic force microscopy images were taken using SPA-400 (SII NanoTechnology Inc., Japan). The silicon cantilever with spring constant $k=12 \mathrm{~N} / \mathrm{m}$ (SI-DF 20; Olympus Corp.) with small tip curvature of less than $10 \mathrm{~nm}$ was used in non-contact mode. All measurements were carried out at room temperature. Surface plasmon resonance (SPR) measurements were carried out using a homemade apparatus, as described elsewhere. ${ }^{20} \mathrm{~A}$ glass substrate with thermally evaporated gold was used as the deposition substrate for SPR measurements.

\section{RESULTS AND DISCUSSION}

\section{Photochemical Control of Surface Wettability Using a Photoreactive Polymer Nanosheet}

The $\mathrm{p}(n \mathrm{PMA} / \mathrm{PhMA})$ copolymer forms a stable and highly oriented monolayer at the air-water interface, as confirmed by

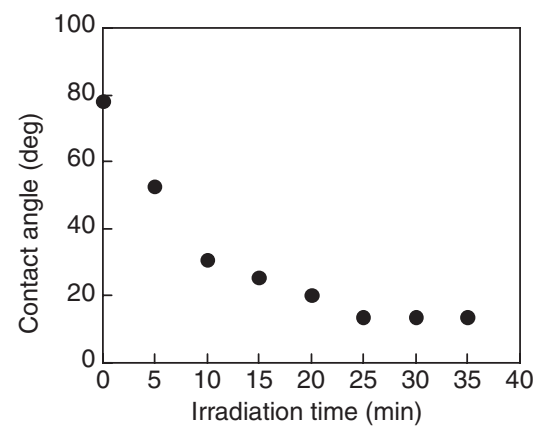

Figure 2. Water CA of a silicon substrate covered with 20 layers of $p(n P M A /$ PhMA) nanosheets with different deep UV irradiation times.

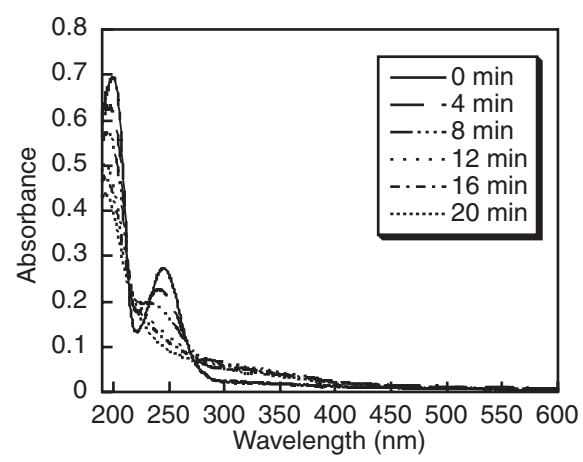

Figure 3. Change in UV absorption spectra of $\mathrm{p}(n \mathrm{PMA} / \mathrm{PhMA})$ nanosheets with deep UV irradiation.

the measurement of $\pi$-A isotherms in the manner reported in a previous study. ${ }^{19}$ In all, 20 layers of $\mathrm{p}(n \mathrm{PMA} / \mathrm{PhMA})$ were deposited onto a silicon substrate. Changes in water contact angles of the photoirradiated $\mathrm{p}(n \mathrm{PMA} / \mathrm{PhMA})$ nanosheets are plotted in Figure 2. Because the $\mathrm{p}(n \mathrm{PMA} / \mathrm{PhMA})$ monolayer forms a highly oriented monolayer at the air-water interface, the surface was occupied by alkyl and phenyl side chains of the polymer. Therefore, the silicon surface covered by $\mathrm{p}(n \mathrm{PMA} /$ PhMA) nanosheets shows large CA of $80^{\circ}$. On the other hand, the water contact angle of the surface decreases gradually with increasing irradiation time (Figure 2) and the water contact angle of the surface covered with the polymer nanosheets was saturated at $14^{\circ}$ after $30 \mathrm{~min}$ irradiation.

We measured UV-vis and FT-IR spectra before and after photoirradiation to elucidate the surface wettability change. Figure 3 portrays the change of UV-visible spectra of 44 layers of $\mathrm{p}(n \mathrm{PMA} / \mathrm{PhMA})$ with deep UV irradiation. The absorbance around $245 \mathrm{~nm}$ and $200 \mathrm{~nm}$, those are attributed to the absorption of phenyl group of PhMA and carbonyl group of PhMA and $n$ PMA respectively, decreases with irradiation time. The UV-vis spectra measurement indicates that photodecomposition of PhMA was carried out by deep UV irradiation. Photochemical reaction in $\mathrm{p}(n \mathrm{PMA} / \mathrm{PhMA})$ was detected by FT-IR.

Figure 4 shows the FT-IR spectra of nanosheets with 40 layers before (Figure 4a) and after (Figure 4b) deep UV irradiation. After photoirradiation, the IR absorption band 


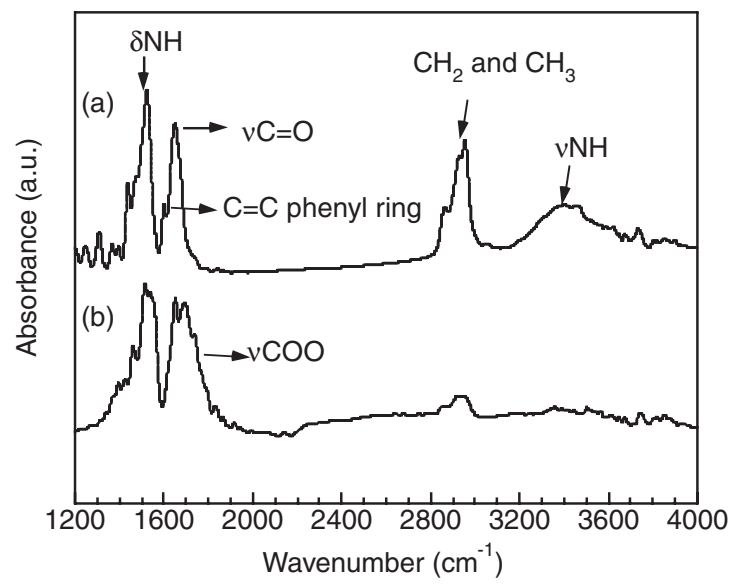

Figure 4. FT-IR spectra of 40 layers of $\mathrm{p}(n \mathrm{PMA} / \mathrm{PhMA})$ nanosheets: (a) before deep UV irradiation and (b) after 30 min deep UV irradiation.

assigned to the alkyl group (2800-3100 $\left.\mathrm{cm}^{-1}\right)$, amide I (1526 $\left.\mathrm{cm}^{-1}\right)$, amide II $\left(1646 \mathrm{~cm}^{-1}\right)$, and phenyl ring $\left(1600 \mathrm{~cm}^{-1}\right)$ were decreased. The broad absorption band assigned to the -NH- group $\left(3400 \mathrm{~cm}^{-1}\right)$ was also decreased. On the other hand, a new band was formed at around $1720 \mathrm{~cm}^{-1}$, which is assigned to $\mathrm{COO}$ of ketone, aldehyde, and carboxylic acid. The decrease in the IR absorption band assigned to alkyl group indicates that $n$ PMA groups were also decomposed during the UV irradiation by the mechanism described below.

The XPS spectrum also indicates the formation of carbonyl groups (Figure 5). The C $1 s$ core-level spectrum of $\mathrm{p}(n \mathrm{PMA} /$ PhMA) nanosheet before photoirradiation can be curve-fitted with components with binding energy at 285.0, 286.0, and 287.9, respectively attributed to $\mathrm{CH}, \mathrm{CN}$, and $\mathrm{OCN}$ groups (Figure 5a). After performing photoirradiation to the $\mathrm{p}(n \mathrm{PMA} /$ PhMA) nanosheet, shoulder peaks appeared at high energy region in the XPS spectrum. The spectrum was fitted with four peaks at $285.1,286.0,288.1$ and $288.8 \mathrm{eV}\left(\chi^{2}=1.3\right)$. These peaks are attributed to $\mathrm{CH}, \mathrm{CN}, \mathrm{OCN}$ and $\mathrm{COO}$ respectively (Figure 5b). Results obtained using UV-vis, FT-IR, and XPS spectra measurements implied that, after photoirradiation, the $\mathrm{p}$ (nPMA/PhMA) was photodecomposed and form carbonyl groups in the polymer. We speculate that the decomposition mechanism proceeds as follows: because phenylacrylamide shows strong absorbance in the deep UV region, the initial decomposition process might be photodecomposition of phenylacrylamide groups. Radical species formed by the photodecomposition react with oxygen to form an active intermediate, such as peroxide radical. ${ }^{21,22}$ Then, the active intermediates react with another site to decompose the alkyl side chains and form oxide species (Scheme 1). A previous report described that direct decomposition of neo-pentylacrylamide groups is negligible in this energy dose. ${ }^{19,23}$

\section{Effect of Film Thickness on the Surface Wettability Change}

Figure 6 shows the contact angle of a silicon substrate covered with different numbers of layers of $\mathrm{p}(n \mathrm{PMA} / \mathrm{PhMA})$ nanosheets before and after deep UV light irradiation. After deposition of two layers of the polymer nanosheets, CA was $80^{\circ}$ because the surface was fully covered by the short alkyl chains and phenyl groups with hydrophobic amide group. The $\mathrm{CA}$ of the polymer nanosheets before photoirradiation was independent of the deposition number of layers, which supports the regular deposition of the polymer nanosheets. Interestingly, after $30 \mathrm{~min}$ photoirradiation, the contact angle decreases gradually with increasing deposition number. The value is saturated to $14^{\circ}$ when the number of layers is greater than 14 layers.

The CA measurements indicate that, with increasing number of layers, many hydrophilic groups were formed after photoirradiation. The $[\mathrm{O}] /[\mathrm{C}]$ ratios obtained from the XPS spectra were used to characterize the degree of surface oxidation (Figure 7). The $[\mathrm{O}] /[\mathrm{C}]$ ratios increase with the increasing number of layers, which shows good agreement with the contact angle measurement results. The contact angle and XPS measurement results indicate that the thicker the nanosheets become, the more $\mathrm{COO}$ groups were produced at the surface of the nanosheets. As described in an earlier chapter, photodecomposition of $\mathrm{p}(n \mathrm{PMA} / \mathrm{PhMA})$ might proceed with the radical chain reaction process. Thicker films have many phenlymethacrylamides; therefore they generate higher concentrations of radicals than the thinner ones after photoirradiation. The generated radical propagates not only through intralayer direction, but also through interlayer direction. Consequently, many oxide species were formed at the surface (a)

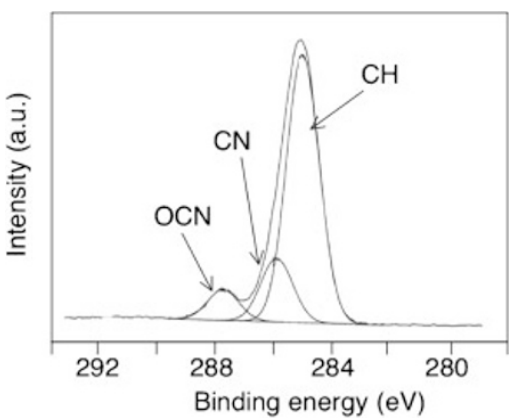

(b)

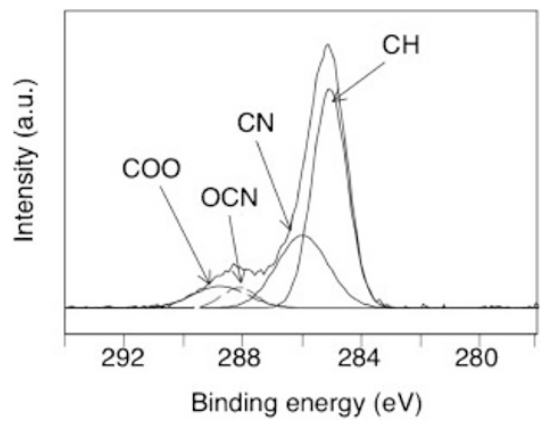

Figure 5. $\mathrm{C}$ 1s core-level XPS spectra of $\mathrm{p}(\mathrm{nPMA} / \mathrm{PhMA})$ nanosheets of 20 layers: (a) before deep UV irradiation and (b) after $30 \mathrm{~min}$ deep UV irradiation. 


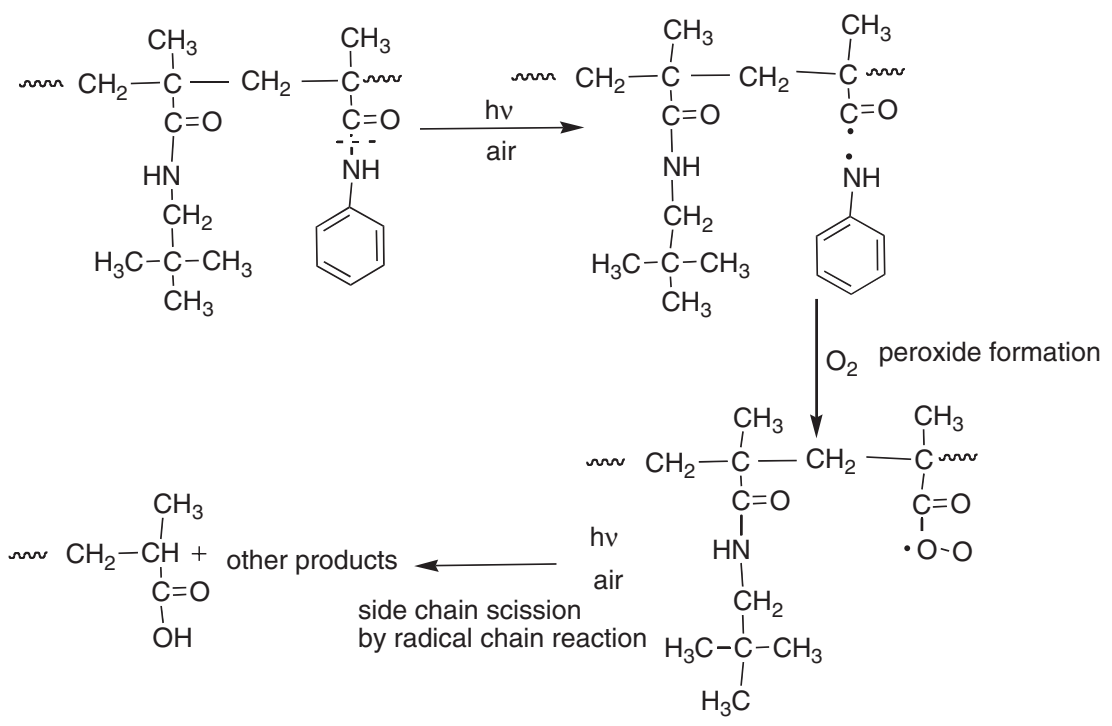

Scheme 1. A possible oxidation scheme of deep UV irradiated $p(n P M A / P h M A)$ nanosheets.

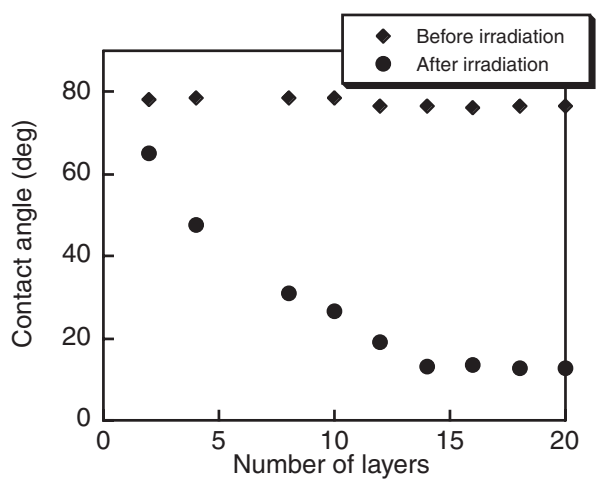

Figure 6. Water $\mathrm{CA}$ of a silicon substrate covered with a $\mathrm{p}(n \mathrm{PMA} / \mathrm{PhMA})$ nanosheet with different number of layers: (a) before UV irradiation and (b) after 30 min deep UV irradiation.

in the thicker film. These results provide sufficient evidence that we can control the wettability of a solid substrate by choosing the number of layers of $\mathrm{p}(n \mathrm{PMA} / \mathrm{PhMA})$.

\section{Surface Morphology and Thickness Measurement}

Surface structure changes before and after photoirradiation of polymer nanosheets were observed using AFM measurements. The initial surface of polymer nanosheets with 20 layers was smooth, with roughness of $1.73 \mathrm{~nm}$ root mean square (Figure 8a). On the other hand, the AFM image of the photoirradiated nanosheet shows several aggregates with height of around $15 \mathrm{~nm}$ and the aggregates distributed on the surface continuously (Figure $8 \mathrm{~b}$ ). The domain was formed by some of photo-cleaved compounds, which were not volatile. The UV absorption around $300 \mathrm{~nm}$ increases with increasing photoirradiation time. Therefore, the non-volatile compounds were probably aromatic compounds formed by a radical coupling of photo-cleaved aniline. Moreover, relaxation of the nanosheets by heat during photoirradiation also induced the morphology change.

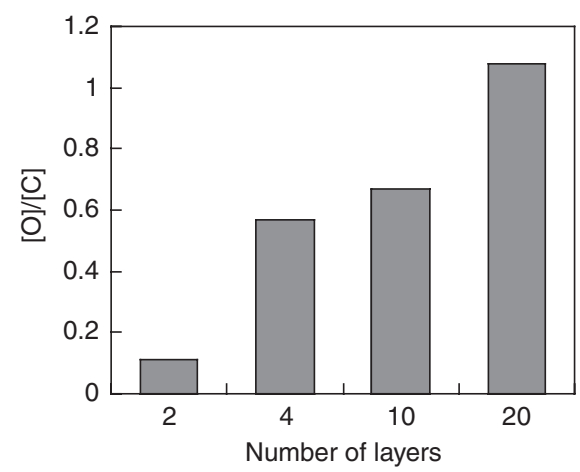

Figure 7. $[\mathrm{O}] /[\mathrm{C}]$ ratio of $\mathrm{p}(n \mathrm{PMA} / \mathrm{PhMA})$ with different numbers of layers after 30 min deep UV irradiation. (a)

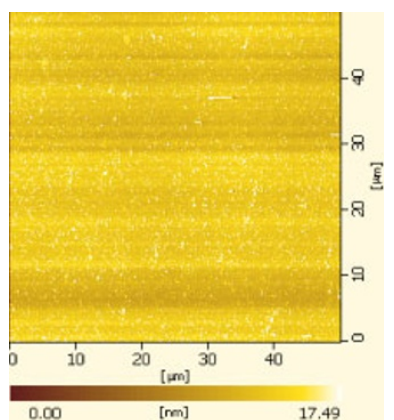

(b)

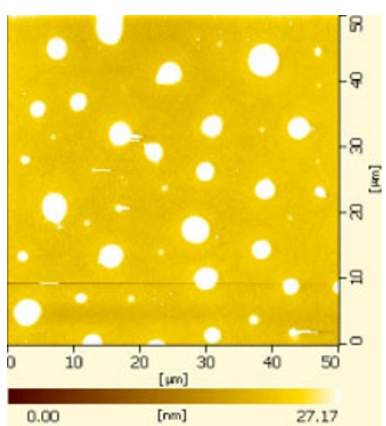

Figure 8. AFM images of $\mathrm{p}(n \mathrm{PMA} / \mathrm{PhMA})$ nanosheets with 20 layers: (a) before deep UV irradiation and (b) after 30 min deep UV irradiation.

Furthermore, the decrease in nanosheet thickness was also observed after photoirradiation. Figure 9 shows SPR spectra before and after photoirradiation with 20 layers. For fitting the SPR spectra, we assumed that the dielectric constant of polymer nanosheets was constant and the polymer nanosheets 


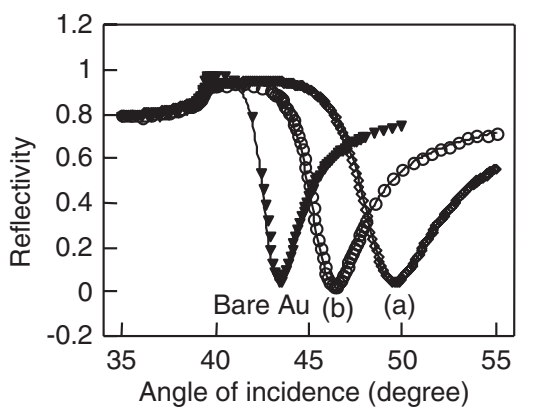

Figure 9. Angle-dependent SPR reflectivity curves of $\mathrm{p}(n \mathrm{PMA} / \mathrm{PhMA})$ nanosheets with 20 layers (a) before deep UV irradiation and (b) after 30 min deep UV irradiation; symbols are experimental points and solid lines are theoretical curves.

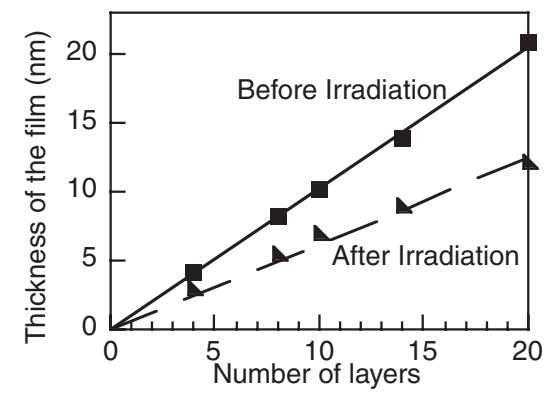

Figure 10. A linear relationship between the thickness and the number of layers before and after deep UV irradiation.

surface was uniformed. ${ }^{24}$ After photoirradiation, the peak position shifted to the smaller angle, which indicates thickness of the film decreased after photoirradiation. The initial thickness of the film increases with increasing layer deposition number, which suggests a regular deposition of the monolayer. The monolayer thickness of the nanosheet was determined as $1.0 \mathrm{~nm}$ from the slope of the straight line (Figure 10), which agrees with the previous value determined using XRD measurements. ${ }^{19}$ After photoirradiation, the film thickness decreased to two-thirds of the initial value, irrespective of the deposition layer number. The thickness decrease after photoirradiation indicates that parts of the polymer were eliminated as volatile products, e.g., $\mathrm{NO}, \mathrm{NO}_{2}, \mathrm{CO}, \mathrm{CO}_{2}$, and $\mathrm{H}_{2} \mathrm{O}$.

\section{CONCLUSION}

A photoreactive polymer nanosheet, $\mathrm{p}(n \mathrm{PMA} / \mathrm{PhMA})$, was used to control the surface wettability by light stimuli. A surface modified with $\mathrm{p}(n \mathrm{PMA} / \mathrm{PhMA})$ nanosheet shows a high contact angle $\left(\mathrm{CA}=80^{\circ}\right)$ because of the alkyl and phenyl side chains of the polymer. After 30 min deep UV irradiation, the water contact angle decreases and becomes hydrophilic $\left(\mathrm{CA}=14^{\circ}\right)$. The surface wettability change was attributed to formation of carbonyl groups at the surface by photodecomposition of the $\mathrm{p}(n \mathrm{PMA} / \mathrm{PhMA})$ nanosheet. Moreover, with increasing thickness of the $\mathrm{p}(n \mathrm{PMA} / \mathrm{PhMA})$ nanosheet, the $\mathrm{CA}$ after UV irradiation decreased and saturated to be $14^{\circ}$ greater than 14 layers. Subsequent XPS measurements revealed that the thicker film contains more oxygen groups than the thinner film; this phenomenon was attributed to a chain reaction of radicals, which forms after photodecomposition of phenylmethacrylamides. This fact implies that the surface wettability can be controlled not only by light irradiation but also by the deposition number of the nanosheets.

Acknowledgment. This research was partially supported using a Grant-in-Aid for Science Research (No. 17105006) from the Ministry of Education, Culture, Sports, Science and Technology of Japan and PRESTO from the Japan Science and Technology Agency.

Received: April 10, 2008 Accepted: June 22, 2008

Published: August 6, 2008

\section{REFERENCES}

1. D. Quéré, Nature Materials, 1, 14 (2002).

2. B. N. J. Persson, O. Albohr, U. Tartaglino, A. I. Volokitin, and E. Tosatti, J. Phys. Condens. Matter, 17, R1 (2005).

3. A. W. Adamson and A. P. Gast, "Physical Chemistry of Surface," 6th ed, Wiley, New York, 1997, Vol. 6.

4. N. Nath and A. Chilkoti, Adv. Mater., 14, 1243 (2002).

5. A. Kikuchi and T. Okano, Prog. Polym. Sci., 27, 1165 (2002).

6. S. Morgenthaler, C. Zink, and N. D. Spencer, Soft Matter, 4, 419 (2008).

7. X. J. Feng and L. Jiang, Adv. Mater., 18, 3063 (2006).

8. A. Ulman, "An Introduction to Ultrathin Organic Films from Langmuir-Blodgett to Self Assembly," Academic press, San Diego, 1991.

9. "Multilayer Thin Films: Sequential Assembly of Nanocomposite Materials," G. Decher and J. B. Schlenoff, Ed., WILEY-VCH, Weinheim, 2003.

10. M. Mitsuishi, J. Matsui, and T. Miyashita, Polym. J., 38, 877 (2006).

11. F. Q. Fan, X. D. Li, and T. Miyashita, Thin Solid Films, 348, 238 (1999).

12. M. Aminuzzaman, Y. Kado, M. Mitsuishi, and T. Miyashita, Polym. J., 35, 785 (2003).

13. F. Q. Fan and T. Miyashita, Polym. J., 33, 857 (2001).

14. C. S. Dulcey, J. H. Georger, V. Krauthamer, D. A. Stenger, T. L. Fare, and J. M. Calvert, Science, 252, 551 (1991).

15. N. Ichinose, H. Sugimura, T. Uchida, N. Shimo, and H. Masuhara, Chem. Lett., 1961 (1993).

16. H. Sugimura, T. Hanji, O. Takai, T. Masuda, and H. Misawa, Electrochim. Acta., 47, 103 (2001).

17. H. Sugimura, K. Ushiyama, A. Hozumi, and O. Takai, Langmuir, 16, 885 (2000).

18. M. Morita, T. Koga, H. Otsuka, and A. Takahara, Langmuir, 21, 911 (2005).

19. Y. Z. Guo, F. Feng, and T. Miyashita, Bull. Chem. Soc. Jpn., 72, 2149 (1999).

20. N. Fukuda, M. Mitsuishi, A. Aoki, and T. Miyashita, J. Phys. Chem. $B$, 106, 7048 (2002).

21. T. Ye, E. A. McArthur, and E. Borguet, J. Phys. Chem. B, 109, 9927 (2005).

22. T. Ye, D. Wynn, R. Dudek, and E. Borguet, Langmuir, 17, 4497 (2001).

23. Y. Z. Guo, F. Feng, and T. Miyashita, Macromolecules, 32, 1115 (1999).

24. M. Mitsuishi, S. Kikuchi, T. Miyashita, and Y. Amao, J. Mater. Chem., 13, 2875 (2003). 